\title{
PROBLEMS IN THE INTERPRETATION OF POLARIZATION MEASUREMENTS IN ACTIVE REGIONS
}

\author{
EBERHARD WIEHR \\ (Göttingen, Universitäts-Sternwarte, Germany)
}

Difficulties and uncertainties are discussed concerning the calibration of the response of a solar vector magnetograph (linear and circular polarization degrees) in terms of field strength $H$ and angle of inclination to the line of sight $\psi$ using the theory of line formation in magnetic fields (Unno, 1956).

For such a calibration two main parameters have to be known quite precisely: $\eta_{0}=\kappa_{\mathrm{c}} / \kappa_{0}$ (the ratio of the absorption coefficient in the line center to the continuous absorption coefficient) and $\beta_{0}$ (the limb-darkening coefficient), both as used in the Milne-Eddington formula for the line profile:

$$
r_{\lambda}=\frac{\beta_{0} \cos \theta}{1+\beta_{0} \cos \theta} \times \frac{\eta_{0} e^{-v^{2}}}{1+\eta_{0} e^{-v^{2}}} .
$$

It is shown that even in the undisturbed photosphere these two parameters are quite uncertain; in a sunspot penumbra or umbra they are practically unknown.

The sensitivity of $H$ and $\psi$ to the choice of $\eta_{0}$ and $\beta_{0}$ has been investigated in detail. Table 1 shows values of $H$ and $\psi$ as obtained from measured polarization degrees $\left(P_{\text {lin }}=1 \% ; P_{\text {circ }}=5 \%\right)$ using different parameters $\eta_{0}$ and $\beta_{0}$ for the Fe line $\lambda 5250 \AA$.

Table 1

$\begin{array}{cccccc} & \beta_{0} & \eta_{0} & \text { profile form } & H \text { (gauss) } & \psi \\ 1 & \gg 1 & 1.5 & \text { numerical } & 970 & 61^{\circ} \\ 2 & \gg 1 & 1.5 & e^{-v^{2}} & 1140 & 61^{\circ} \\ 3 & 2 & 10 & e^{-v^{2}} & 680 & 45^{\circ} \\ 4 & \gg 1 & 1.5 & \text { numerical with } & 1130 & 63^{\circ} \\ & & & 20 \% \text { straylight } & & \end{array}$

All profiles are normalized to an equivalent width $W_{\lambda}=70 \mathrm{~m} \AA$ and central depth $r_{\mathrm{c}}=0.6$.

These computations show that in general it is not possible to determine the magnetic-field structure with a desirable accuracy even from very precise polarization measurements. Therefore a vector magnetograph has to be calibrated empirically. 
This has been done recently at the Crimean Astrophysical Observatory (Severny, 1967).

Details will be published elsewhere in connection with the final results of measurements obtained in active regions on March 21-29 and May 20-29, 1967.

\section{References}

Unno, W. (1956) Publ. astr. Soc. Japan, 8, 108.

Severny, A. B. (1967) Izv. Krym. astrofiz. Obs., 36, 22.

\section{DISCUSSION}

Maltby: I think that this paper brings out very clearly the need for a method of the type developed by Kjeldseth Moe, where the depth dependence of the line-absorption coefficient is taken into account.

Wiehr: Generally I agree with you, and I am looking forward for this paper. On the other side the difficulties originating from the depth dependence of the magnetic field and of the choice of the model of the various parts of an active region will remain.

Mattig: You have spoken about the strong depth dependence of the absorption coefficient for the magnetic sensitive line $\lambda 5250 \AA$, especially in sunspots. I want to say that in sunspots the absorption coefficient $\eta$ for the iron line $\lambda 6303 \AA$ is nearly independent from optical depth. This is a result of new calculations with different sunspot models. So I think it is better to observe in the red than in the green line, if you want to interpret the magnetograms using the Unno theory.

Wiehr: The red line is difficult to use, because of the terrestrial blend on the decreased sensitivity of the multiplier. On the other hand, the depth dependence of the absorption coefficient can be calculated by the Moe method, as Dr Maltby pointed out. But here also one needs a model, which is unknown especially for the penumbra, where a vector magnetograph is most useful - in the photosphere the fields are too small, in the umbra one has saturation effects. 